\title{
Fusion of Biogeography based optimization and Artificial bee colony for identification of Natural Terrain Features
}

\author{
Priya Arora \\ Mtech student, CSE \\ RIEIT, PTU \\ Punjab, India
}

\author{
Harish Kundra \\ HOD, CSE \\ RIEIT, PTU \\ Punjab, India
}

\author{
Dr. V.K Panchal \\ Associa te Director, Sci 'G' \\ DTRL, DRDO \\ Delhi, India
}

\begin{abstract}
Swarm Intelligence techniques expedite the configuration and collimation of the remarkable ability of group members to reason and learn in an environment of contingency and corrigendum from their peers by sharing information. This paper introduces a novel approach of fusion of two intelligent techniques generally to augment the performance of a single intelligent technique by means of information sharing. Biogeography-based optimization (BBO) is a recently developed heuristic algorithm, which proves to be a strong entrant in swarm intelligence with the encouraging and consistent performance. But, as BBO lacks inbuilt property of clustering, its behavior can be replaced with the honey bees of artificial bee colony (ABC), a new swarm intelligent technique. These two methods can be combined to create a new method which is easy to implement and gives more optimized results than the results when $\mathrm{BBO}$ is used. We have successfully applied this fusion of techniques for classifying diversified land cover areas in a multispectral remote sensing satellite image. The results illustrate that the proposed approach is very efficient than BBO and highly accurate land cover features can be extracted by using this approach.
\end{abstract}

Keywords- Biogeography-based Optimization; Artificial bee colony; Hybrid swarm intelligence; Image classification; Multi spectral dataset.

\section{INTRODUCTION}

Swarm Intelligence is an venture to design algorithms or distributed problem solving methods stimulated by collective behavior of social insect colonies. Examples of systems studied by Swarm Intelligence are colonies of ants and termites, schools of fish, flock of birds, herds of land animals. Various Swarm based approaches have been developed till now. A novel approach added recently in this category called Biogeography-based Optimization proposed by Dan Simon (2008) [1]. Karaboga and Basturk (2005), proposed artificial bee colony algorithm for solving many optimization problems which is easy to implement and robust. The key to sustaining global, self- organized behavior is social interaction.

The fundamental principle of these techniques is cooperation and sharing of knowledge. The basic of the increased intelligence is the shared information discovered individually and communicated to the swarm by different mechanisms of social interactions. In this paper, a novel integrated SI algorithm is developed by synthesizing Biogeography based optimization [2] and artificial bee colony optimization [3]. We have tried to make the BBO technique more intelligent simply by making the species in BBO to work according to the honey bees of $\mathrm{ABC}$. In this way, intelligent solutions to problems naturally will emerge from the selforganization and communication among simple individuals. To validate the effectiveness of the proposed approach, it is applied for the classification of various natural terrain features which itself is an optimization problem. We have compared our results with $\mathrm{BBO}$ to show the performance of our proposed technique.

In this paper, the first section is an introduction to swarm intelligence. The second section gives detail of the BBO and $\mathrm{ABC}$. The third section discusses the proposed approach i.e. Fusion of $\mathrm{BBO}$ and $\mathrm{ABC}$. The fourth section discusses the implementation of the proposed concept for classifying natural terrain features. The last section presents the concluding remarks.

\section{BACKGROUND}

\section{A. Biogeography Based Optimization}

The idea of Biogeography Based Optimization (BBO) was first presented in December 2008 by D. Simon[2]. Biogeography is the study of the geographical distribution of biological organisms. The mindset of the engineer is that we can learn from nature. This motivates the application of biogeography to optimization problems. Just as the mathematics of biological genetics inspired the development of genetic algorithms (GAs), and the mathematics of biological neurons inspired the development of artificial neural networks, mathematics of biogeography as the basis for the development of a new field: biogeography-based optimization (BBO).

Mathematical models of biogeography describe how species migrate from one island to another, how new species arise, and how species become extinct. The species are migrated to the suitable habitats i.e, feature islands. These habitats are actually the decisions, for the resultant groups. Various methods decide which species are moved to which habitat using a fitness function [2]. The fitness function is actually the information that is shared among all the habitats in order to decide the suitable habitat for migration of each species. Geographical areas that are well suitable as residences 
for the biological species are said to have a high suitability index (HSI) [2]. Features that correlate with HSI include factors such as rainfall, diversity of vegetation, land area and temperature. The variables characterize habitability are called suitability index Variables (SIVs). SIVs can be considered the independent variables of the habitat, and HSI can be considered as the dependent variable.

Habitats with a high HSI have many species that emigrate to nearby habitats, simply by the virtue of the large number of species that they host. Habitats with a high HSI have a low species immigration rate because they are already nearly saturated with species. Habitats with a low HSI have a high species immigration rate because of their sparse populations. Biogeography is nature's way of distributing species and is analogous to general problem solutions. Suppose that we are presented with a problem and some candidate solutions. A good solution is analogous to an island with a high HSI and a poor solution rep resent an island with a low HSI. We call this approach to problem solving Biogeography Based Optimization [1].

\section{B. Artificial Bee Colony}

ABC was originally proposed by Dervis Karaboga [3] under the inspiration of collective behavior of honey bees. Real bee colony is one of the natural societies with most specialized social behaviors. ABC algorithm consists of three kinds of bees: employed bees, onlooker bees, and the scout bees[5]. The goal of the whole colony is to maximize the amount of nectar.

1. Employed bees determine a food source considering the surrounding food source in their memories.

2. Employed bees inform the onlooker bees in the hive about the food source and onlooker bees select a food source.

3. Employed bee whose source is abandoned becomes a scout bee and start to search for a new food source.

All the information about the currently rich food source are available on the dance floor area and the onlooker watches numerous dances performed by the employed bees and chooses the profitable food source. The onlooker bee decides the profit using the probability values of the food sources. The recruitment is thus proportional to the profitability of a food source. Basically, there are two important functions that supports the algorithm[5].

$$
\begin{gathered}
P_{i}=\frac{f i t_{i}}{\sum_{n=1}^{S N} f i t_{i}} \\
\mathbf{V}_{\mathrm{ij}}=\mathbf{X}_{\mathrm{ij}}+\Phi_{\mathrm{ij}}\left(\mathbf{X}_{\mathrm{ij}}-\mathbf{X}_{\mathbf{k j}}\right)
\end{gathered}
$$

where $P_{i}$ is the probability value associated with $\mathbf{i}_{\text {th }}$ food source that calculated by the Eq a. An onlooker bee selects a food source relying on $\mathrm{P}_{\mathrm{i}}$. In this equation, fit $\boldsymbol{i}_{\boldsymbol{i}}$ represents $\mathbf{i}_{\text {th }}$ food sources nectar amounts, which is measured by employed bees and $\mathrm{SN}$ is the number of food source which is equal to the number of employed bees. Fitness is calculated using following equation;

$$
f i t n e s s(i)=\left[\begin{array}{cc}
1 /\left(1+f n c_{i}\right), & f n c_{i} \geq 0 \\
1+a b s\left(f n c_{i}\right), & f n c_{i}<0
\end{array}\right]
$$

the $\boldsymbol{f n c}_{\boldsymbol{i}}$ in the equation is the cost function of the quality of source. Greedy selection is applied to select the best source. In the real world problems, $\mathbf{X}_{\mathbf{i j}}$ and $\mathbf{X}_{\mathbf{k j}}$ represents the different old food source positions. The difference between these two positions is the distance from one food source to the other one. $\mathrm{D}$ is number of optimization parameters. $\varphi_{\mathrm{ij}}$ is a random number between $[-1,1]$ and controls the distance of a neighbor food source position around $\mathbf{X}_{\mathbf{i j}}[5]$.

\section{INTEGRATED ALGORITHM BASED ON BBO AND ABC}

$\mathrm{BBO}$ and $\mathrm{ABC}$ are two outstanding swarm intelligence methods. Biogeography describes how various species migrate from one island to another according to their suitability conditions. The heuristic method decides which species are moved to which habitat using a fitness function. The fitness function is actually the information that is shared among all the habitats in order to decide the suitable habitat for migration of each species. In $\mathrm{ABC}$ the bees distributes themselves to various food locations and finally on finding most suitable food source the bees are followed by rest of their bee mates to that particular food source. In our proposed technique we have replaced the behavior of species in BBO by the behavior of honey bees of $\mathrm{ABC}$.

The main idea of this proposed approach is to enhance the quality of $\mathrm{BBO}$ by bartering information from the other intelligent technique i.e. ABC. Thus the species will be randomly distributed to the habitats rather than moving with the whole group of species. All the featured habitats will be explored by the species. On finding a habitat whose HSI is near about the defined fitness value, neighborhood of that specific habitat will be further explored by the species in order to find a better place to migrate. Thus on finding a habitat whose HSI is high, the species will exchange the information of that habitat with the rest of the species waiting for them (Universal habitat) as the employed bees exchange information related to different food sources with the onlooker bees. Now the whole group will collectively migrate to that suitable habitat for their migration.

In this way we can create knowledge by sharing information between two intelligent techniques to make a technique more intelligent. Hence this proposed intelligent computing hybrid approach would be able to solve complex problems providing more efficient results which would be better than the results of $\mathrm{BBO}$. The framework of the algorithm could be described as follows :

\section{Initialize solutions belongs to different habitats.}

2. Select species from the universal habitat and randomly distribute the species to the feature habitats (It will be executed according to the employed bees' search)

3. Calculate the HSI (fitness value) for each habitat.

4. If the HSI calculated is within the threshold value then explore the neighborhood solution else go to step 2 . 
5. Select the most suitable habitat for migration.

6. End.

In order to validate this proposed scheme, it will be experimented on a set of data.

\section{EXPERIMENT SETUP}

We have applied this fusion of $\mathrm{BBO}$ and $\mathrm{ACO}$ on the remote sensing multi- spectral, multi resolution and multi sensor image of Alwar area in Rajasthan with dimensions 472 * 572 for classifying the various terrain features. The area is selected since it contains a good variety of land use features like urban, water body, rocky, vegetation and barren areas. The 4 spectral bands are in the visible bands namely : red, green, near - infrared (NIR), and middle infra - red (MIR) from LISS- 111 sensor of Indian Remote Sensing sat satellite Resource sat -1 . RS1 and RS2 are the bands of the same area taken from Canadian Radarsat-1 satellite. The seventh image is digital elevation model (DEM) of the area. The ground resolution of these images from LISS-111 and Radarsat-1 images are $23.5 \mathrm{~m}$ and $10 \mathrm{~m}$ respectively. The DEM dataset is also generated from SAR interferometer using RS1 and RS2 and have 25 -meter resolution and is taken from LISS $=11$, sensor and is provided to us courtesy of DTRL(Remote Sensing) Lab, DRDO, Delhi. The 7 band Satellite image of Alwar Area in Rajasthan is given in figure 1.
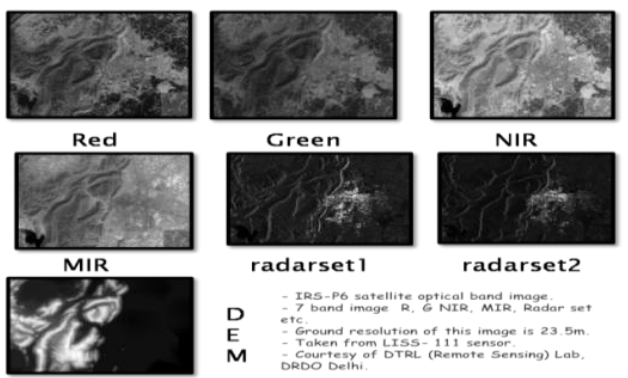

radarset 1

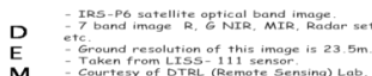

Figure1. 7-Band Satellite Image of Alwar region, Rajasthan.

\section{A. Proposed Algorithm for classification}

Assumptions

Food sources are the land cover features- water, vegetation, urban, rocky, and barren.

Input :Dataset of Multi- spectral satellite image.Output : Classified image.

\section{Step 1: Initialization}

Consider original image as species of universal habitat.
Consider each feature class as one habitat. Total no of habitat $=$ Universal + Feature habitat .

Step 2: Allocation of species according to employed bees.

- Distribute species to various habitats (according to employed bees' search)

Step 3 : Calculate HSI of each habitat.

- Evaluate HSI of each habitat here calculated using Mean of Standard Deviation as

$$
H S I=\sum_{k=1}^{m b} S(i, k) / m b
$$

where $\mathrm{i}$ is for each habitat (different feature exist on the study area), varies from 1 to $\mathrm{mb}$.

$\mathrm{S}_{\mathrm{ik}}$ represents Standard Deviation of $k$ th band of $i$ th habitat ( $\mathrm{k}$ varies for each availasble band, i.e. 1 to $\mathrm{mb}$, where $\mathrm{mb}$ is the number of band available)

$$
S_{i, k}=\frac{\sqrt{\sum_{j=1}^{n_{i}} V_{i}(k, j)-M(i, k)}}{n_{i}}
$$

where

$>\mathbf{n}_{\mathbf{i}}$ is the training data available for each land cover type.

$>\mathrm{V}_{\mathrm{i}}(\mathrm{k}, \mathrm{j})$ represents the pixel value of $\boldsymbol{k}$ th band in $\boldsymbol{j t h}$ training pixel of $i$ th habitat.

$>\mathrm{M}_{\mathrm{i}, \mathrm{k}}$ is the mean of $\boldsymbol{k}$ th band in the $i$ th habitat

Step 4: Selection of Habitat

- If calculated HSI is within threshold then

$>$ Continue searching the neighborhood of that habitat for better solutions.

$>$ Absorb species to that habitat.

- else

$$
\text { go to step } 2 .
$$

- Check for the other habitats and recalculate the HSI.

- End loop when all pixels classified.

- Display the output classified image.

B. Flowchart 


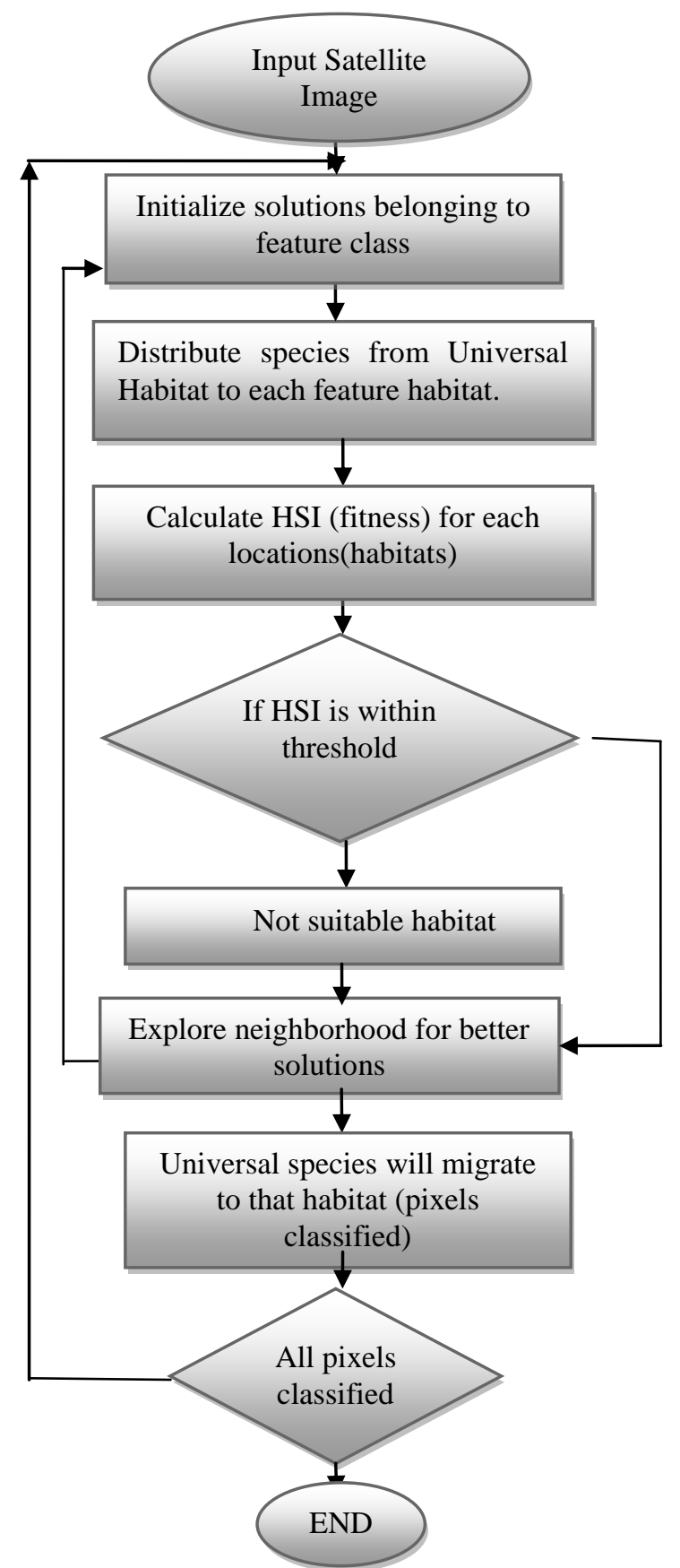

Figure 3 shows the satellite false color image and Figure 4 is the classified image of Alwar region after applying BBO$\mathrm{ABC}$.
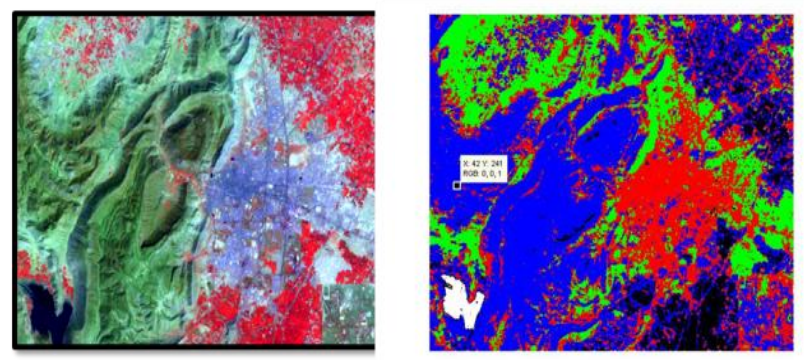

Figure 3. ORiginal Image Figure 4. Classified IMAge Table Type

\section{Accuracy statement}

A classification is not complete until its accuracy is assessed [6]. Practically it is not feasible to test every pixel of a class known. Instead a set of reference pixels is used. These are the points on the classified image for which actual features are known. The reference pixels are randomly selected. The main goal of accuracy assessment is to quantitatively determine how effectively pixelare grouped into the correct feature classes in the area under investigation. In our proposed work the classification accuracy is expressed using classification error matrix. For the validation process we have taken into consideration following number of pixels;

Table1. Error Matrix when BBO -ABC is applied

\begin{tabular}{|l|l|l|l|l|l|l|}
\hline Feature & Water & Veg & Urban & Rocky & Barren & Total \\
\hline Water & 68 & 0 & 0 & 0 & 0 & 68 \\
\hline Veg & 0 & 109 & 1 & 0 & 0 & 110 \\
\hline Urban & 0 & 0 & 112 & 0 & 4 & 116 \\
\hline Rocky & 0 & 0 & 0 & 96 & 0 & 96 \\
\hline Barren & 0 & 0 & 26 & 0 & 59 & 85 \\
\hline Total & 68 & 109 & 139 & 96 & 63 & 475 \\
\hline
\end{tabular}

- 68 water pixels.

- 109 vegetation pixels.

- 139 urban pixels.

- 96 rocky pixels.

- 63 barren pixels.

The Kappa coefficient of the Alwar image can be calculated by applying following formula to the Error Matrix;

$$
\hat{k}=\frac{N \sum_{i=1}^{r} x_{i i}-\sum_{i=1}^{r}\left(x_{i+} \cdot x_{+i}\right)}{N^{2}-\sum_{i=1}^{r}\left(x_{i+} \cdot x_{+i}\right)}
$$

- $r=$ number of rows in the error matrix $(r=5$ in our case)

- $\quad \mathrm{X}_{\mathrm{ii}}=$ the number of observations in row $\mathrm{i}$ and column $i$ (on the major diagonal)

- $\mathrm{x}_{\mathrm{i}+}=$ total no of observation in row $\mathrm{i}$ (shown as marginal total to right of the matrix)

- $\mathrm{x}_{+\mathrm{i}}=$ total of observations in column $\mathrm{i}$ (shown as marginal total at bottom of matrix)

- $\mathrm{N}=$ total number of observations included in matrix. $(\mathrm{N}=475$ in our case $)$

The Kappa coefficient of the Alwar image is 0.917, which indicates that an observed classification is 91.7 percent better than one resulting from chance whereas the value of Kappa coefficient in case of BBO is 0.69121 [4]. So it is clearly understood that our classifier has good classification accuracy as compared to $\mathrm{BBO}$. 


\section{CONCLUSION AND FUTURE WORK}

This paper introduces the fusion of two swarm intelligent techniques $\mathrm{BBO}$ and $\mathrm{ABC}$. The particles in a swarm try to solve a problem as a group by using the information contained by their peer mates. This sharing of information enables swarm particles to be more efficient and to achieve goals that they could not achieve individually. To check the efficiency of our proposed approach it has been used for the classification of natural terrain features. Experimentally we have proved that the proposed algorithm yields an approximate but usually good solution to the classification problem for satellite images. The kappa coefficient of 0.917 also supports our algorithm efficiency from our table which are better than the results when BBO whose kappa coefficient is 0.69121 . We can say that for the given dataset we have been able to classify water, vegetation, rocky areas perfectly. Therefore we illustrate the information sharing concept in the swarm intelligence techniques by means of a terrain understanding application. In future, the concept of information sharing can be exploited further to adapt to other applications such as groundwater exploration through case based reasoning using any of the above SI techniques. This practice can be easily being extended for other global optimization problems.

\section{REFERENCES}

[1] Ralph W. Kiefer, Thomes M. Lillesand, "Principles of Remote Sensing",2006.

[2] Dan Simon, "Biogeography Based Optimization", IEEE transactions on evolutionary computation, vol. 12, no. 6, December 2008
[3] D Karaboga , " An idea based on honey bee swarm for numerical optimization TR-06", 2005.

[4] V.K Panchal, D Bhugra, S Goel, V Singhania, " Study On The Behaviour Of BBO Over Natural Terrain Features", Electronics Computer Technology (ICECT), 3rd International Conference,2011.

[5] Yuk Ying Chung, Wei-Chang Yeh, Noorhaniza Wahid, Ahmad Mujahid, Ahmad Zaidi, "Artificial Bee colony based data mining Algorithms for classification task",vol 5, no. 6; August 2011.

[6] T.MLillesand, R.W Keifer, J.W Chipman, "Remote Sensing and Interpretation", New York: John Wiley \& Sons Ltd, pp, 586 -592(2004).

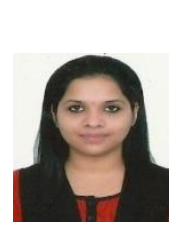

AUTHORS PROFILE

Priya Arora has done B-Tech (Hons) in Information Technology \& scored $78 \%$ marks from Punjab Technical University, Jalandhar (India) in 2010 and Mtech in CSE from Punjab Technical University with $70 \%$ marks.

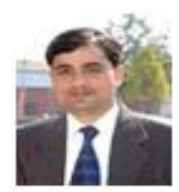

Harish Kundra is an Assistant Professor and Head of Department in CSE \& IT Department of Rayat Institute of Engineering \&IT, Railmajra, Punjab, India. He has presented 15 papers in International/National journals. He is a lifetime member of Indian Society of Technical Education.

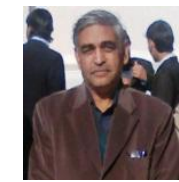

V.K Panchal is Associate Director at Defence Terrain Research Lab, New Delhi, Associate Member of IEEE, (Computer Society) and Life Member of Indian Society of Remote Sensing. Chaired sessions \& delivered invited talks at many national \& international conferences. Research interest are in synthesis of terrain understanding model based on incomplete information set using bio-inspired intelligence and remote sensing. 